\title{
EL CONTEXTO SOCIOHISTÓRICO DE LAS INVESTIGACIONES DE SAMUEL K. LOTHROP EN EL DELTA DEL PARANÁ, ARGENTINA
}

\author{
THE SOCIO-HISTORICAL CONTEXT OF SAMUEL K. LOTHROP'S \\ RESEARCH IN THE PARANÁ DELTA, ARGENTINA
}

\author{
Mariano Bonomo ${ }^{1}$ y Máximo Farro ${ }^{2}$
}

\begin{abstract}
En este artículo se estudia la historia de las investigaciones de Samuel Kirkland Lothrop, Jr. (1892-1965) en el Delta del Paraná, Argentina. Sus objetivos son (i) contextualizar los trabajos de campo en los sitios arqueológicos Arroyo Malo, El Cerrillo y Arroyo Sarandí, (ii) aportar a la historia de la disciplina y (iii) analizar las redes establecidas entre las instituciones involucradas (Museo de La Plata y Museum of American Indian). Para ello se relevaron documentos inéditos y bibliografía sobre este arqueólogo norteamericano, los cuales revelan que durante las dos guerras mundiales Lothrop actuó como espía en distintos países de Latinoamérica. Palabras claves: nordeste argentino, Museo de La Plata, Museum of American Indian, trabajo de campo, redes de informantes, espionaje.
\end{abstract}

This article explores the history of Samuel Kirkland Lothrop, Jr.'s research in the Paraná Delta of Argentina (1892-1965). The objectives are threefold: (i) to contextualize his archaeological fieldwork at Arroyo Malo, El Cerrillo and Arroyo Sarandí, (ii) to contribute to the history of the discipline, and (iii) to analyze the networks created among the institutions involved (i.e., Museo de La Plata and The National Museum of The American Indian). Toward these purposes, Lothrop's unpublished documents and bibliography were brought together and analyzed, revealing that during the two World Wars, Lothrop acted as a spy in various Latin American countries.

Key words: Argentinean Northeast, Museo de La Plata, National Museum of the American Indian, fieldwork, correspondent networks, espionage.

Este artículo presenta un estudio de la historia de las investigaciones de Samuel K. Lothrop en el Delta del Paraná, Argentina. Su objetivo específico es contextualizar históricamente los trabajos de campo de Lothrop (1932) en los sitios arqueológicos Arroyo Malo, El Cerrillo y Arroyo Sarandí. Más allá de contribuir a la memoria histórica de nuestra disciplina en Latinoamérica, en estas páginas se reflejan las diferentes implicancias sociales de la práctica arqueológica y de las relaciones institucionales que permean cotidianamente el desarrollo de las investigaciones en ese campo. El caso de Lothrop es un claro ejemplo de cómo la reconstrucción del pasado puede estar atravesada por objetivos económicos o políticos e incluso por las estrategias militares del momento. Las importantes investigaciones arqueológicas efectuadas en el Delta del Paraná por Samuel Lothrop, favorecidas por su visión global del pasado americano, han dejado una ineludible impronta en la arqueología rioplatense hasta el día de hoy. Las investigaciones del arqueólogo norteamericano en el Delta del Paraná tuvieron y tienen un fuerte impacto en la arqueología pampeana, del Nordeste argentino, de Uruguay y el sur de Brasil.

Para este trabajo fueron consultados artículos periodísticos, fotografías, cartas, el diario y las notas de campo depositados en el archivo Samuel K. Lothrop del Peabody Museum of Archaeology and Ethnology (PMAE) de Harvard University (Cambridge, Massachusetts), institución para la cual Lothrop trabajó cuando aún era estudiante de grado y donde retornó e investigó hasta sus últimos días como emérito (Willey 1976). Específicamente se analizó la documentación disponible en la colección Lothrop Papers \# 996-20 y 996-21 (carpetas 9, 10, 14, 15, 16 y 18: Journal, field notebook, correspondence, photographs, loose notes and newspaper scraps). Además se estudió el material del Archivo Histórico del Museo de La Plata (AHMLP), específicamente los Libros copiadores

\footnotetext{
1 CONICET, División Arqueología del Museo de La Plata, Facultad de Ciencias Naturales y Museo, UNLP, La Plata, Argentina. mbonomo@fcnym.unlp.edu.ar

2 CONICET, Archivo Histórico del Museo de La Plata, Facultad de Ciencias Naturales y Museo, UNLP, La Plata, Argentina. mfarro@fcnym.unlp.edu.ar
} 
de correspondencia (LC) y las Carpetas de correspondencia, Dirección y Secretaría del Instituto del Museo de La Plata (IMD).

\section{La Expedición Thea Heye-Museo de La Plata}

Durante la década de 1920 tuvieron lugar en la Argentina una serie de expediciones de carácter paleontológico y arqueológico, sufragadas por instituciones norteamericanas, que contaron con el apoyo, asesoramiento y control legal de sus actividades por parte de los principales museos del país: Museo de Ciencias Naturales de Buenos Aires, Museo Etnográfico y Museo de La Plata. Como caso particular, la expedición arqueológica al Delta del Paraná de 1925, formó parte de una empresa cooperativa entre esta última institución, dirigida desde 1920 por Luis María Torres, y el Museum of American Indian-Heye Foundation, con sede en Nueva York, creado y dirigido por el empresario George Gustav Heye en 1916.

En esos años, Torres (Figura 1) había reorientado los objetivos del Museo que desde 1906 formaba parte de la Universidad Nacional de La Plata, adjuntando a las funciones de exhibición y estudio de las colecciones, las de enseñanza académica. Torres recupera parte del programa fundacional de la institución, creada por Francisco P. Moreno en 1884, que colocaba el énfasis en las exploraciones científicas del territorio argentino (Farro 2009; García 2010). Por otro lado, en esos años había establecido también relaciones de colaboración para solventar los gastos en las exhibiciones, las colecciones y la adquisición de obras bibliográficas, con acaudalados mecenas como Victoria Aguirre o el empresario Benjamín Muniz Barreto, coleccionista de piezas arqueológicas del Perú y del Noroeste argentino, quienes donaban periódicamente sumas de dinero (Farro et al. 2012; García 2010).

Por su parte, Heye, como muchos otros empresarios norteamericanos de las primeras décadas del siglo XX (Snead 1999), se dedicaría a armar colecciones de objetos principalmente arqueológicos y, en menor medida etnográficos, centrándose exclusivamente en el continente americano, montando una colección que para 1906 contaba con 10.000 objetos, procedentes de Nuevo México y Arizona. Para aumentar su volumen, si bien compró pequeñas series de objetos a indígenas y a coleccionistas locales, Heye adquirió grandes lotes de materiales, principalmente colecciones etnográficas

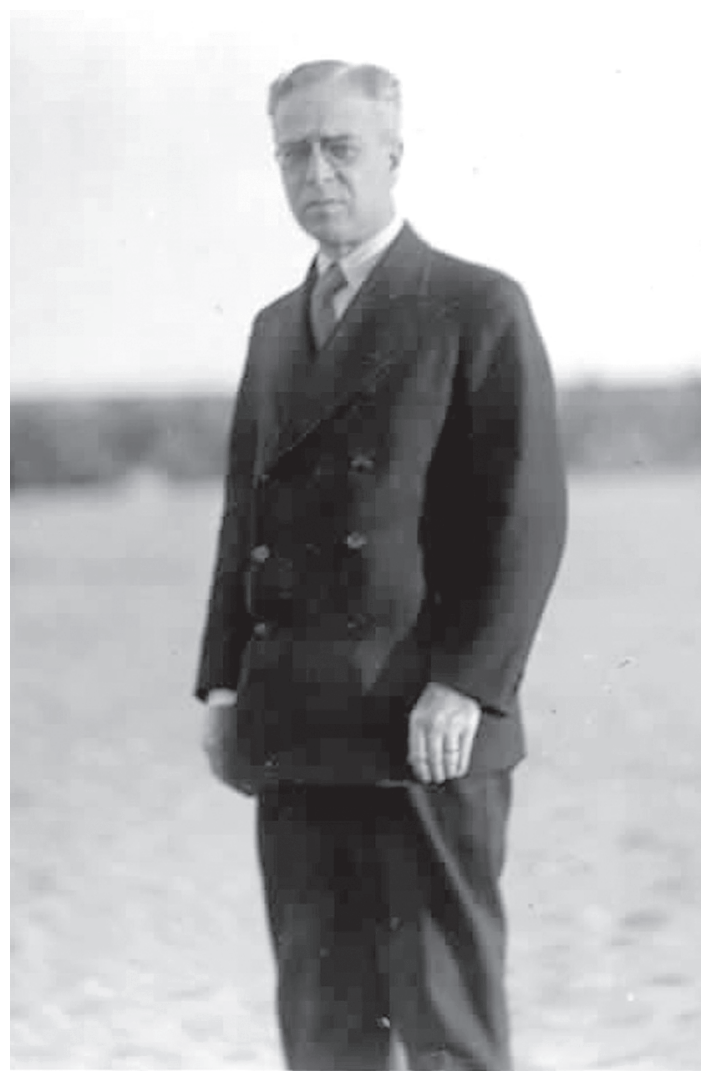

Figura 1. Luis María Torres, director del Museo de La Plata. Luis María Torres, Museo de La Plata's director.

en las principales firmas proveedoras de Londres y París, lo cual constituyó un hecho particular en la época (Jacknis 2006). En el caso de las colecciones arqueológicas, sufragó los gastos de expediciones y publicaciones, asesorado por dos arqueólogos discípulos de Frederick W. Putnam: Marshall H. Saville (1867-1935), de Columbia University, y George Pepper, del American Museum of Natural History. Ambos actuaron como curadores-asistentes de sus colecciones, recomendándole las maneras apropiadas de registro de los objetos y las prácticas adecuadas para el montaje de series sistemáticas (Lothrop 1957; Jacknis 2006). En 1916, con la ayuda financiera de acaudalados amigos, fundó el Museum of American Indian del que fue nombrado director perpetuo, donando su colección particular que por entonces se componía de unas 58.000 piezas.

Cabe destacar que ya desde 1904 Heye había puesto en marcha un plan de expansión de las colecciones, dirigido e ideado por Saville, cuyo objetivo era la obtención de series arqueológicas y etnográficas a lo largo del continente americano. Esto incluía, 
en primer lugar, la realización de expediciones dirigidas por miembros del staff de la institución, principalmente en el Sudoeste norteamericano, Alaska, Canadá, México (Michoacán), Puerto Rico, Ecuador (Manabí) y las Antillas. Con ese fin, Heye contrató en 1918 a Frederick W. Hodge, miembro del Bureau of American Ethnology, y en 1924 a Samuel Kirkland Lothrop (Figura 2), egresado de Harvard en 1921 y especializado en arqueología de Mesoamérica. En segundo lugar, ese plan incluía un programa de canje de colecciones con otras instituciones, que se hizo especialmente activo en vísperas de la apertura del museo al público en 1922, con el objetivo de contar con colecciones representativas de todo el continente. Precisamente en ese momento, Randolph Saville, hijo de Marshall, durante un viaje a la Argentina visitó el Museo de La Plata, haciendo la propuesta inicial de un canje de piezas de arqueología del Noroeste argentino, en especial de objetos de cobre, bronce y cerámica, a cambio de una colección de valor equivalente del Ecuador. En esa ocasión Saville también había cerrado

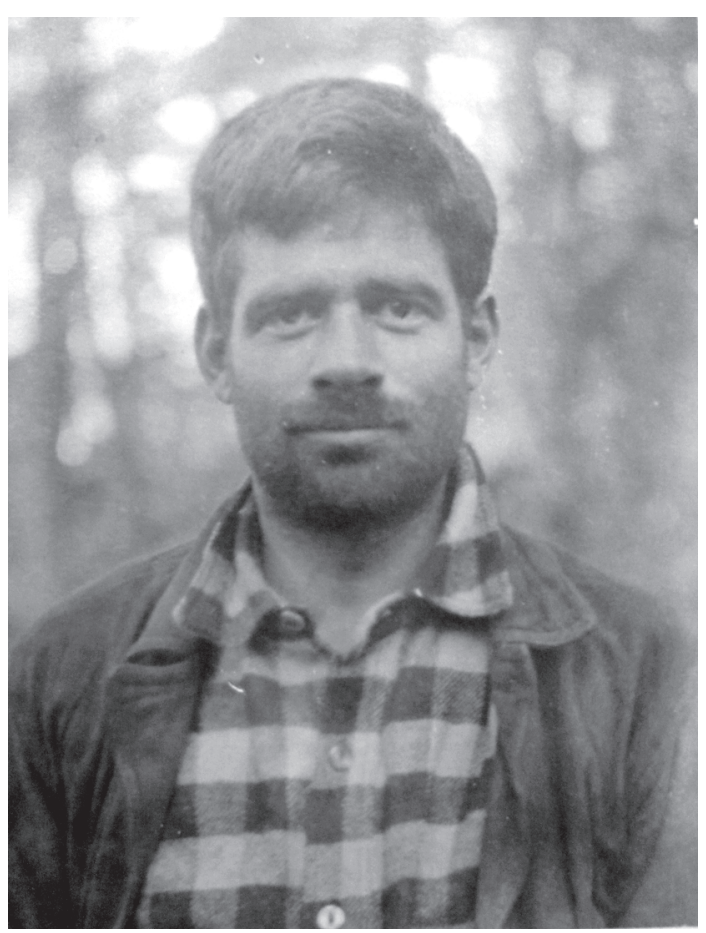

Figura 2. Samuel K. Lothrop en Harberton (Tierra del Fuego, Argentina, 1925) [Copyright 2014 President and Fellows of Harvard College, Peabody Museum number 2004.1.132.104]. Samuel K. Lothrop in Harberton (Tierra del Fuego, Argentina, 1925) [Copyright 2014 President and Fellows of Harvard College, Peabody Museum number 2004.1.132.104]. un acuerdo de canje con el Museo Etnográfico de Buenos Aires ${ }^{1}$. Con posterioridad, Marshall ofrecerá cincuenta piezas, entre cerámica y hachas de piedra del Ecuador, solicitándole a Torres el envío de colecciones arqueológicas y etnográficas más numerosas y de otros puntos del país, para poder obtener así series más representativas ${ }^{2}$. Por último, a mediados de 1924, durante un viaje a la Argentina, Heye y su mujer Thea Page se entrevistaron con Torres en el Museo de La Plata, ofreciendo una colección mayor a la pautada originalmente, en condiciones ventajosas para la institución argentina, ya que solventarían todos los gastos de envío de lo intercambiado ${ }^{3}$. Unos meses más tarde, el matrimonio Heye decidió estrechar los lazos de cooperación de ambas instituciones, proponiendo el desarrollo conjunto de una expedición arqueológica, dirigida por Lothrop, financiando todos los gastos que la misma requiriese y dividiendo las colecciones obtenidas en partes iguales ${ }^{4}$. El director del Museo de La Plata aceptó el ofrecimiento y decidió realizar la expedición en el Delta del río Paraná, donde él había trabajado desde 1905 (Márquez Miranda 1937; Torres 1911) $)^{5}$.

La expedición estaba programada para comenzar el 1 de marzo de 1925, inmediatamente después de finalizada una expedición dirigida por Lothrop en Tierra del Fuego, iniciada en diciembre del año anterior (Lothrop 1925b). A pesar de las gestiones anticipadas del arqueólogo norteamericano y de las promesas de Torres, el inicio de los trabajos en el Delta del Paraná sería retrasado en varias oportunidades debido a la gestión administrativa de los permisos para excavar y para la exportación de colecciones de carácter arqueológico y antropológico, ante el Ministerio de Instrucción Pública de la Nación ${ }^{6}$. La gestión de los permisos se enmarcaba en la reglamentación que se había establecido en 1921 de la ley 9080 de protección de los yacimientos antropológicos, arqueológicos y paleontológicos, sancionada en 1913, que originalmente declaraba la propiedad de la Nación sobre los mismos, quedando las colecciones obtenidas en la esfera del dominio público, en detrimento del derecho de propiedad de los particulares (Endere 2000; Endere y Podgorny 1997). Los permisos de excavación se concedían a instituciones científicas del país o del extranjero que realizaran investigaciones, sin fines comerciales, quedando en exclusividad para las primeras los derechos de excavación sobre yacimientos de carácter excepcional. La gestión de los permisos y los 
trabajos de explotación estaba a cargo de la Sección o "Comisión de Yacimientos" cuya presidencia, de régimen anual, se ejercía de manera alternada entre el Museo de Ciencias Naturales de Buenos Aires, el Museo Etnográfico y el Museo de La Plata (Farro et al. 2012). Entre otras cosas, esa comisión debía llevar un registro de los lugares a ser explorados, el personal científico que interviniera en cada caso y el número de piezas recogidas por la misión autorizada. También debía anotar en un mapa especial todos los yacimientos numerándolos ordinalmente, a lo que debía agregarse en cada caso una carta topográfica bien delimitada del lugar a explorar. Por su parte, los jefes de las misiones científicas autorizadas tenían que tomar nota de todos los trabajos que realizaran en el terreno, determinando las formaciones geológicas y clasificando todos los materiales hallados, inventariándolos para dejar a su regreso una copia de todo en la mencionada comisión 7 .

Para promocionar y hacer aplicable en la práctica a la ley 9080 la estrategia seguida por Torres incluía, por un lado, la utilización de la administración del aparato escolar nacional y provincial, solicitando en este caso a los directivos y maestras el envío de cartas de aviso en caso de hallazgos arqueológicos y de fósiles (García 2010). Por otro, en llevar a la práctica una estrategia similar a la seguida por Francisco P. Moreno a fines del siglo XIX, como era el envío de circulares y de una copia adjunta de la mencionada ley a los gobernadores de las provincias y territorios nacionales de Argentina solicitándoles la prioridad en la comunicación de la identificación por parte de los residentes locales de enterratorios indígenas y huesos paleontológicos (Farro 2009) ${ }^{8}$. En ese sentido, la ley pareció apuntar, más que al control de las actividades de los coleccionistas, aficionados y de los proveedores locales que vendían objetos al mejor postor, a la regulación de las actividades de las misiones científicas extranjeras y a la salida de piezas fuera del país (Farro et al. 2012). En efecto, a pesar de las gestiones anticipadas de Lothrop y Heye y de las promesas de Torres al respecto, el inicio de los trabajos en el Delta del Paraná sería retrasado en varias ocasiones alegando cuestiones burocráticas 9 . Los permisos se retrasaron también por una cláusula objetable para los norteamericanos en el convenio establecido entre las instituciones, referido a la dirección total de la expedición, ya que Lothrop solicitó abrogarse en exclusiva la dirección de los trabajos en el campo, tal como había establecido como condición Mr. Heye en una carta a Torres, que Lothrop le entregó personalmente, fechada el 9 de octubre de $1924^{10}$.

Ante el retraso en los permisos, Lothrop acudió a sus contactos personales con llegada a los ámbitos de decisión ministeriales de la Argentina, como Kenneth Apollonio, miembro como él de la cohorte 1915 de Harvard University, residente en Buenos Aires y dedicado a los negocios de importación con la firma Apollonio \& Co., representante en el país de las corporaciones norteamericanas más importantes (Harvard Alumni Association 1926). Es interesante destacar aquí, en primer lugar, el creciente peso de las filiales empresarias norteamericanas en la gestión de las expediciones desarrolladas por instituciones de ese país en la Argentina desde el cambio de siglo (Podgorny 2005), en un contexto de fuerte presencia en el mercado local, por medio de un agresivo proceso de inversión directa que tuvo su auge a lo largo de la década de 1920 (Lanciotti y Lluch 2009). Esto incluía el envío de giros, la gestión de la correspondencia, los instrumentos de trabajo y movilidad, el embalaje y el envío de las colecciones resultantes. Así, parte de la gestión administrativa de las expediciones paleontológicas del Field Museum comandadas por Elmer Samuel Riggs en el sur de la provincia de Buenos Aires, Catamarca y la Patagonia entre 1923 y 1927, estuvo a cargo de la empresa frigorífica Swift del Plata \& $\mathrm{Co}^{11}$, estando a su vez la logística del trabajo de campo montada sobre la infraestructura que la compañía Standard Oil había desarrollado para la explotación de hidrocarburos en la Argentina (Thorne y Thorne 1995). En el caso de la expedición de Lothrop, Heye había arreglado la gestión de la misma con el gerente de la filial argentina de The American Express Company Inc ${ }^{12}$. En segundo lugar, la estrategia seguida por Lothrop revela el peso que seguían teniendo aun en las primeras décadas del siglo XX los vínculos personales en la gestión de las expediciones. En este sentido, Lothrop poseía un nutrido capital relacional, derivado de las actividades comerciales de su padre en Centroamérica y de sus años como estudiante universitario (Easby 1965; Willey 1976).

\section{Desarrollo de la Expedición}

Como consta en el diario y en el cuaderno de notas, los trabajos de campo en el Delta del Paraná $^{13}$ se llevaron a cabo entre el 6 de abril y el 
11 de junio de 1925. Allí Lothrop excavó los sitios arqueológicos Arroyo Malo, El Cerrillo y Arroyo Sarandí (Figura 3), localizados en los ambientes insulares y litorales del Paraná Inferior (Lothrop 1925a, 1932:124). Los trabajos de excavación se insertaban en los estándares científicos de la época, caracterizados por la apertura a pala de trincheras por medio de seis peones contratados. El estipendio por día de los peones era de seis pesos, más un peso extra por cada vasija intacta, 25 centavos por las rotas y 50 centavos por las hachas de piedra. Como aliciente extra, a esto se le podían agregar cinco pesos para aquel que encontrara una canoa como la que había hallado en las vísperas de la expedición Pablo Gaggero, Jefe de Trabajos Prácticos de Zoología y Biología en la Escuela de Ciencias Naturales del Instituto del Museo de La Plata, en el lecho del arroyo La Glorieta (llamado arroyo Rodeo por Lothrop) y que había sido adquirida para esa institución. Esta canoa, hecha sobre un tronco de timbó cuyo interior había sido quemado, había sido encontrada en las cercanías del sitio Arroyo Malo ${ }^{14}$.

Colaboraron en las investigaciones Rachel Warren, su primera esposa, y Antonio Castro, preparador de la institución platense contratado para tareas especializadas: ambos lavaban y clasificaban los materiales arqueológicos extraídos por los peones, empacaban los objetos en bolsas de papel y luego en cajones de madera para su transporte en barco. Simultáneamente, Lothrop llevaba un cuaderno de notas y un diario de campo, y dibujaba con lápiz y acuarelas los planos de los sitios, levantados con brújula y pasos, y también diseñaba las vasijas enteras, los tiestos con decoración incisa y las disposiciones de los entierros humanos. Los controles periódicos por parte de la "Comisión de Yacimientos" incidieron también sobre las tareas de registro de la información, ya que Lothrop, al no tener certeza sobre el destino final de cierta clase de objetos hallados que, por su carácter único, con probabilidad serían reclamados para engrosar las colecciones de la institución de $\mathrm{La}$ Plata, tomó la precaución de dibujarlos detalladamente (PMAE, Lothrop Journal -de aquí en adelante LJ-, May $15^{\text {th }}$, p. 52).

Este registro basado en sustratos escritos era acompañado por el registro fotográfico de los paisajes y la vida isleña, los campamentos de campaña, las tareas de excavación y los materiales arqueológicos más destacados. Lothrop y su esposa además se ocupaban de cuestiones logísticas, como la compra

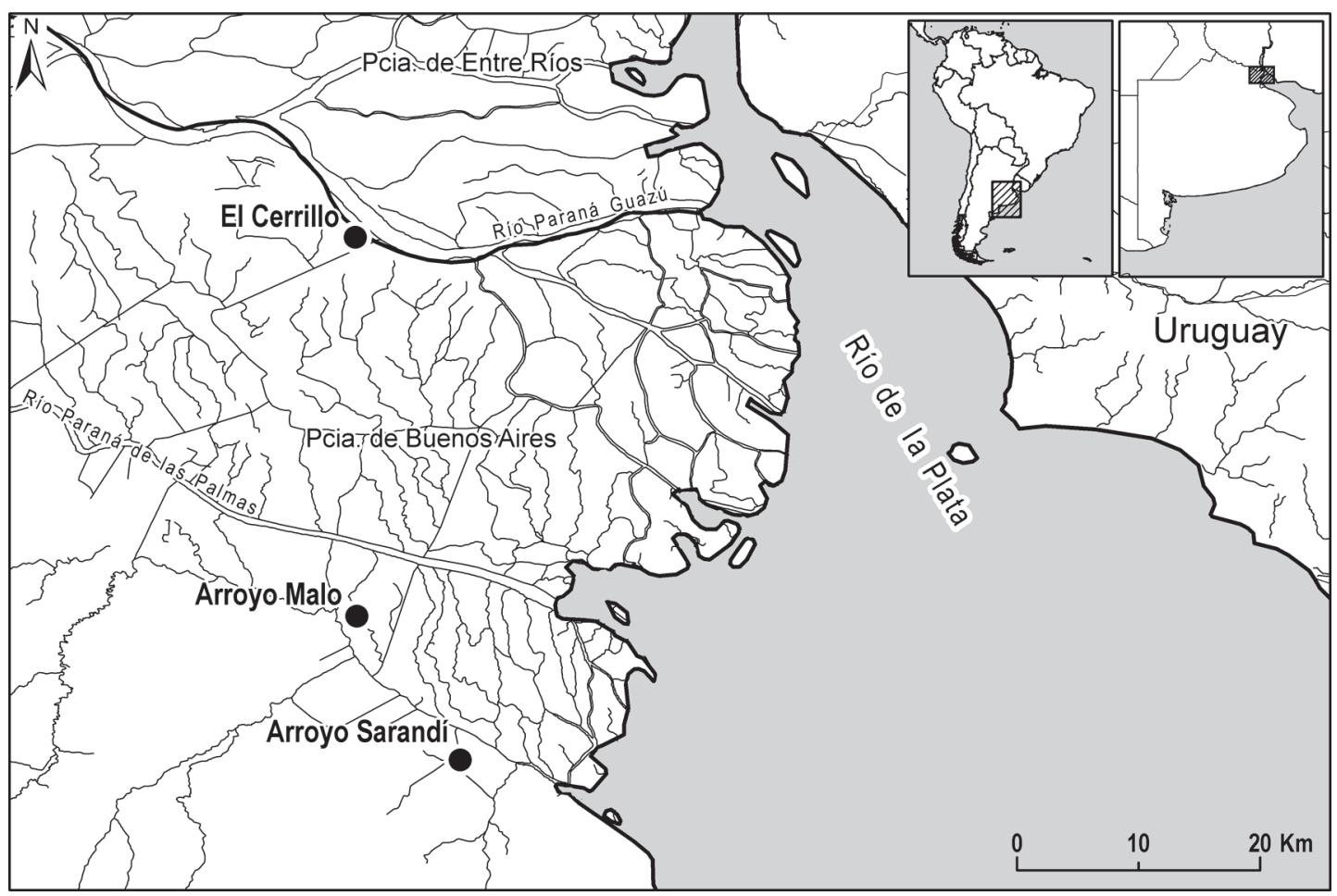

Figura 3. Localización geográfica de los sitios arqueológicos Arroyo Malo, El Cerrillo y Arroyo Sarandí. Geographic location of Arroyo Malo, El Cerrillo and Arroyo Sarandí archaeological sites. 
de herramientas o el abastecimiento de comida. Para ello contaron con la colaboración de informantes locales, como del Jefe de Gendarmería de Islas ${ }^{15}$ quien envió un sargento al que se le asignó la función de cocinero. Pero sobre todo contaron con el apoyo de un colaborador del Museo de La Plata en la región, el descendiente de italianos Eugenio Tricerri, residente en la isla Pampero, quien puso a disposición de los Lothrop su propia casa para establecer la base general de operaciones de la expedición. En los hechos, el apoyo implicaba la puesta a disposición de una nutrida red de contactos en las islas, indispensables para obtener rápidamente los permisos de excavación en las fincas, la gestión de la correspondencia, los giros, el depósito y transporte de objetos y personal, la contratación de peones, la comunicación de hallazgos o la obtención de materiales de trabajo, como herramientas adecuadas y $\operatorname{carpas}^{16}$. En numerosas ocasiones el éxito completo de una expedición dependía de la labor de estos corresponsales (Farro 2009).

Arroyo Malo fue el primero de los sitios trabajados, en las proximidades de la quinta de Domingo Novellino, en la isla Pampero, donde el matrimonio Lothrop estableció su campamento, acompañados por Apollonio. El sitio arqueológico estaba localizado en la isla adyacente llamada "La Sirena", frente al canal Gobernador Arias, en el albardón de la margen derecha del arroyo Guayracá, conocido en el lugar como arroyo Malo. Por ello diariamente para trasladarse hasta el sitio tenían que usar una embarcación y luego seguir a pie entre zanjas y pajonales. Los datos sobre este y otros sitios para excavar fueron brindados a Lothrop por Gaggero. En febrero de 1925 Gaggero, quien a su vez tuvo noticias de Arroyo Malo por aviso de Tricerri, había pasado tres semanas en el sitio donde constató que los pobladores habían extraído varias urnas y él mismo exhumó otras más ${ }^{17}$. Las urnas, que estaban en su mayoría fragmentadas a causa del arado y las raíces de los árboles, poseían huesos humanos pintados de rojo y una vasija pequeña en su interior (véase también Vignati 1941). A los pocos días que Lothrop había comenzado con los trabajos allí, Gaggero lo visitó junto a Castro; este último se iba a integrar rápidamente al equipo de excavación provocando una excelente impresión en el norteamericano por su habilidad y voluntad de trabajo. El desarrollo de la expedición y de los trabajos de excavación y extracción de materiales era inspeccionado por Torres periódicamente, con el objeto de verificar que se cumplieran las cláusulas establecidas por la "Comisión de Yacimientos" para las expediciones extranjeras. Varios pasajes del diario de Lothrop permiten apreciar cómo funcionaban en la práctica los trabajos de control y la manera en que estos incidían en el ritmo de las tareas, en la posibilidad de acceder a determinados sitios y en el tipo de objetos que podían ser llevados fuera del país (LJ, April 25th , p. 28; May ${ }^{\text {st }}$, pp. 34-35).

Arroyo Malo fue clasificado por Lothrop como una aldea perteneciente a los guaraníes, grupos agricultores de origen amazónico que llegaron al Delta del Paraná alrededor de dos siglos antes que los conquistadores europeos. Esta atribución del sitio a los guaraníes sigue vigente en la actualidad y se basaba en la presencia de entierros humanos en urnas, hachas de piedra pulida, platos, escudillas, tinajas y ollas de cerámica polícroma y corrugada.

El 31 de abril, al finalizar la campaña en Arroyo Malo, y debido a un retraso de diez días en el permiso para trabajar en Arroyo Sarandí, Lothrop siguió la recomendación de Torres y, mientras se solucionaba el problema burocrático, buscó un sitio alternativo para excavar en el río Paraná Guazú, donde gracias a la mediación de Tricerri con los propietarios de la zona, consiguió rápidamente el acceso a las fincas para excavar un sitio (LJ, May $3^{r d}$, p. 36). Dado que el lugar para acampar se encontraba en muy malas condiciones y alejado del sector a excavar, se trasladó a otro sitio que denominó El Cerrillo (Figura 4), trabajando allí desde el 11 al 24 de mayo. Este sitio, localizado en la margen derecha del río Paraná Guazú entre los cursos Paraná Miní y canal Segunda Campana, ofreció grandes dificultades para el trabajo por las constantes crecidas que inundaban las cuadrículas abiertas, impidiendo las actividades de los peones. Durante la excavación lo visitó nuevamente Torres, quien le señaló que en realidad estaba trabajando en el "Túmulo I" del Paraná Guazú (Figura 5), montículo que él mismo ya había investigado en $1905^{18}$ (Lothrop 1932:146-148; Torres 1911), lo que generó ciertas tensiones, ya que el director del Museo de La Plata lo creía totalmente agotado (LJ, May $21^{\text {th }}$ and $22^{\text {th }}$, pp. 61-62). Torres solicitó para las colecciones del Museo los veintitrés esqueletos extraídos y las series de restos animales. Los abundantes tiestos cerámicos lisos e incisos recuperados por Lothrop, más una interesante colección de instrumentos óseos (puntas, cabezales de arpones y tubos) y líticos (punta de proyectil, bola de boleadora, materiales 


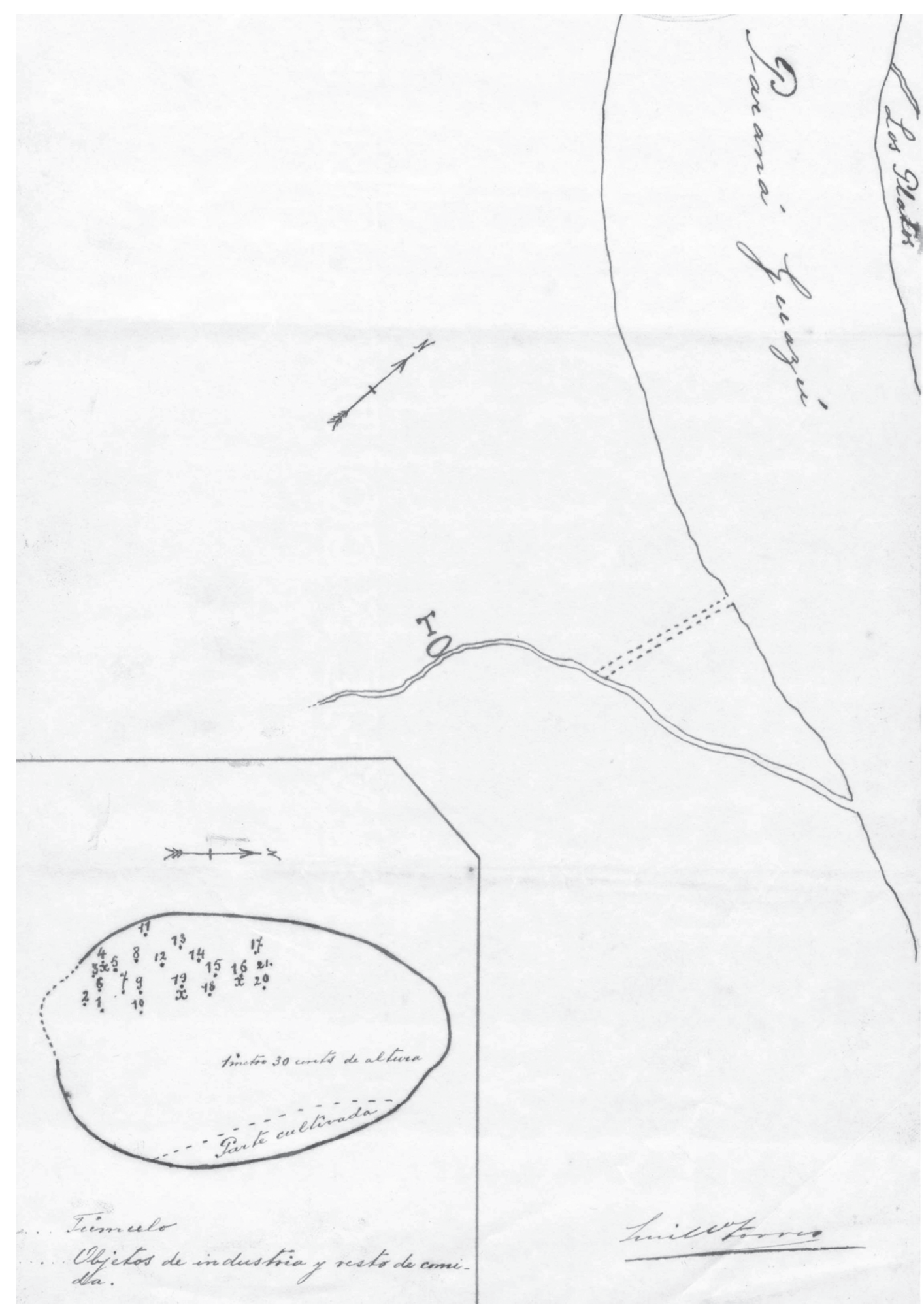

Figura 4. Ubicación de los sitios arqueológicos excavados en el Delta del Paraná en 1905, expedición del Museo de La Plata. Croquis realizado por Luis María Torres.

The archaeological sites excavated in the Paraná Delta, 1905, Museo de La Plata expedition. Sketch made by Luis María Torres. 

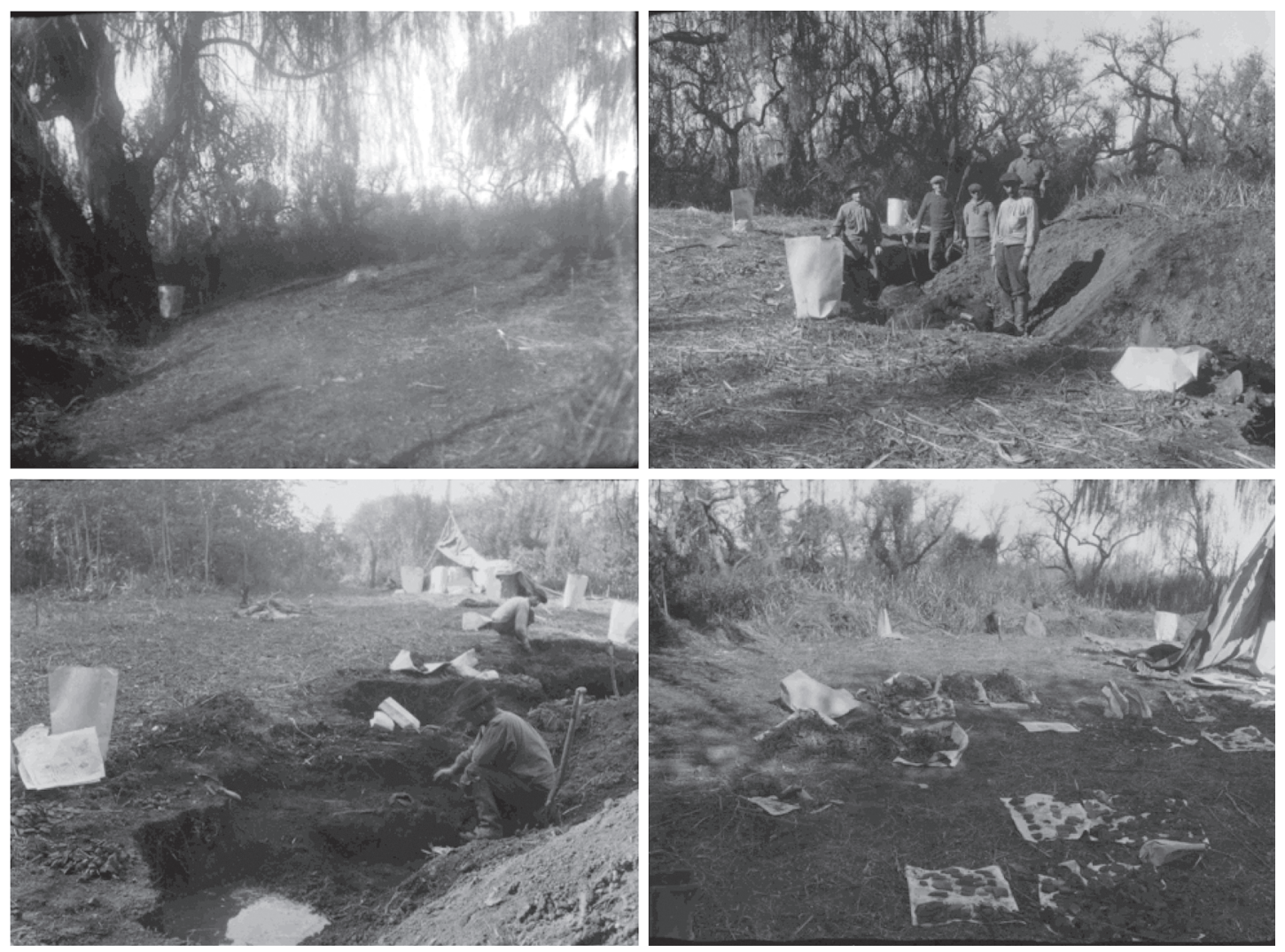

Figura 5. Trabajo de campo de los Lothrop en El Cerrillo [National Museum of American Indian, Smithsonian Institution, números de catálogo: N10477, N10479, N10480, N10483].

Lothrop's fieldwork in El Cerrillo [National Museum of American Indian, Smithsonian Institution, catalog numbers: N10477, N10479, N10480, N10483].

de molienda, percutores y yunques) fueron enviados a Norteamérica. De acuerdo con su ubicación geográfica y a los restos recuperados este sitio fue atribuido por Lothrop a los grupos étnicos locales denominados chaná o chaná-mbeguá.

Por último, luego de una espera de más de un mes por la gestión por parte de Torres de los permisos necesarios, Lothrop se trasladó finalmente al campo Pacheco, en el Tigre, donde se encuentra el Arroyo Sarandí (margen sur del río Luján), trabajando allí entre el 1 y el 11 de junio, momento en que las excavaciones debieron finalizarse por el comienzo de las frías heladas. Este sitio, compuesto por dos elevaciones, A y B, que Lothrop llamó "mounds" y Castro "túmulos" (según figura en las etiquetas de las colecciones depositadas en el MLP), estaba emplazado en un lugar mucho más accesible que aquellos puntos de las islas del Paraná excavados antes, en una zona densamente atravesada por las infraestructuras del comercio y el turismo. Esto provocaba numerosas visitas a las excavaciones de paseantes de fin de semana y de los reporteros gráficos de los principales diarios del país, como "Crítica" y "La Razón". Más aún, esta relación con el mundo del turismo y la recreación se puede observar en un dato en apariencia menor: en la publicación oficial con los resultados de la expedición, para dar cuenta de la ubicación de los sitios trabajados Lothrop utilizó de base un mapa de la región publicado por la afamada marca de vermouth "Cinzano" que aparecía intercalada en la "Guía Tigre" (véase Fig. 6, en Lothrop 1932:124). Si en las dos excavaciones anteriores los Lothrop debieron lidiar con las roturas y alteraciones provocadas por las inevitables visitas de los familiares y amigos de sus colaboradores locales, en este caso, dado el volumen de turistas, prepararon el sitio con sogas alrededor de las cuadrículas con la finalidad de que la gente no rompiera ni robara nada, en un momento donde llegaron a recibir la visita de entre 300 y 400 personas en un solo día (LJ, April 14 ${ }^{\text {th }}$, p. 17). 
Uno de los atractivos reseñados por los medios gráficos eran los esqueletos hallados (Figura 6), atribuidos a los cazadores-recolectores querandíes, que fueron puestos al descubierto por el arado (Crítica, 9 y 10 de junio de 1925). Estos esqueletos habían sido pedidos por Torres cuando visitó Arroyo Sarandí y le ofreció otro sitio arqueológico para que Lothrop excavara en el Delta, pero este último se negó dado que ya había cumplido con su objetivo y no necesitaban más piezas en Nueva York. En Arroyo Sarandí se hallaron abundantes materiales cerámicos (cuenco, tortero, alfarerías tubulares y pendientes), materiales líticos (disco auricular, bolas, puntas de proyectil, materiales de molienda, percutores y yunques), cuentas sobre valvas e instrumentos óseos (puntas, biseles, ganchos de propulsor y horquetas perforadas). En el Museo de La Plata también quedaron depositados algunos instrumentos óseos de Arroyo Sarandí y alfarería, artefactos líticos y objetos de origen europeo de Arroyo Malo, parte de los cuales fueron recientemente localizados y reestudiados (Bonomo et al. 2009).

\section{Lothrop, el Trabajo de Campo y las Redes de Espionaje}

Al llegar a la Argentina, Lothrop ya poseía una importante experiencia profesional, lograda a partir de investigaciones arqueológicas en distintos países americanos: Puerto Rico, Guatemala, Costa Rica, Honduras, Panamá, El Salvador, México (Yucatán) y Estados Unidos (La Nación, 8 de abril de 1925; Easby 1965; Willey 1976). Cabe destacar aquí que esta nutrida experiencia en la dirección de trabajos de campo arqueológicos en América Central sirvió en la práctica como camuflaje para otra actividad en la que se desempeñó activamente a lo largo de toda su carrera profesional: el espionaje. Como otros arqueólogos y antropólogos estadounidenses, británicos y alemanes lo hicieron a lo largo del siglo
XX en distintas partes de América, Europa, Asia y África (Allen 2011; Price 2008; Richter 2008), Lothrop estuvo involucrado en tareas de inteligencia y espionaje con objetivos político-militares.

Durante la Primera Guerra Mundial Lothrop se desempeñó como miembro activo del U.S. Office of Naval Intelligence (ONI), a la que también perteneció su primera mujer, Rachel Warren, que actuó en varias ocasiones como agente encubierta (Browman 2011; Harris y Sadler 2003; Price 2008). A partir de la completa investigación de Harris y Sadler (2003) podemos conocer los detalles de las actividades de Lothrop durante la Primera Guerra: gracias a las gestiones de su amigo el arqueólogo Sylvanus Morley, entre mayo de 1917 y agosto de 1918, fue reclutado como agente civil del ONI, donde ejerció como el Agente Especial № 173 y Rachel Warren como el Agente S-32, ambos encargados de operativos de espionaje en el área de América Central. Samuel, cuyo seudónimo para los cables secretos era "Laposso", actuó principalmente en Nicaragua, Costa Rica y el Canal de Panamá por lo que recibía un salario mensual (Harris y Sadler 2003). A partir de una serie de controversias y denuncias en su contra, Lothrop fue exonerado y se le ordenó regresar de inmediato a Estados Unidos en agosto de 1918 dado que su identidad como agente de inteligencia había sido revelada. No obstante, rápidamente comenzó una breve carrera militar y entre octubre de 1918 y marzo de 1919 fue nombrado segundo teniente del ejército estadounidense, asignado a tareas de inteligencia en el extranjero (Harris y Sadler 2003). Después de eso fue puesto con un estatus voluntario dentro de la ONI en calidad de "Reserve Officer" (Price 2008).

En estos tiempos de guerra el matrimonio Lothrop participó activamente del mundo del espionaje y se involucró en numerosos operativos de inteligencia. Lothrop formó una red de subagentes asalariados para recabar información política y

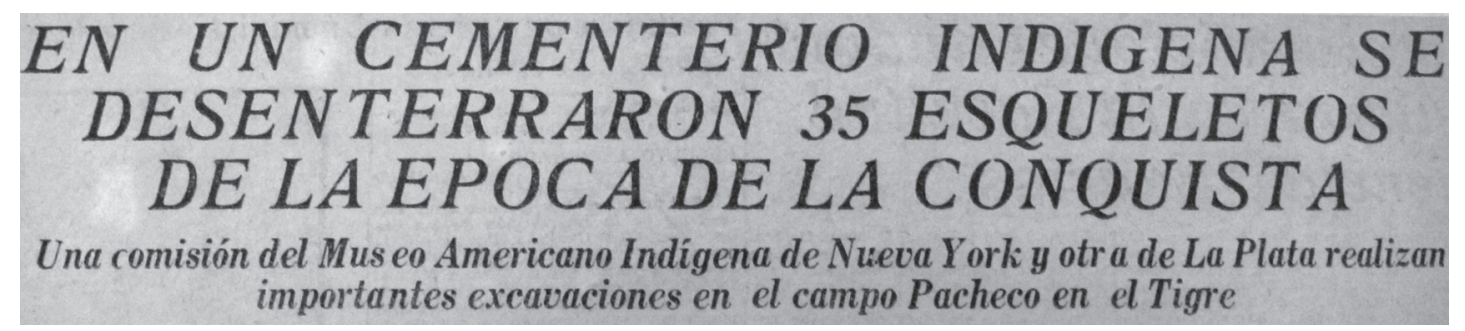

Figura 6. Titular de la nota del diario Crítica referido a los trabajos de los Lothrop en el Delta del Paraná, 9 de junio de 1925. Headline from the newspaper "Crítica" about the Lothrops' fieldwork in the Paraná Delta, June 9, 1925. 
comercial dentro del Distrito Naval a su cargo. Utilizó para ello los libros de la ONI, encriptando datos por medio de códigos especiales diseñados para sustituir las letras y los números, que llegaban en valijas diplomáticas estadounidenses, y enviando correos confidenciales utilizando la infraestructura comunicacional de las empresas de su país en la región, como la United Fruit \& Co. También levantó planos de posibles puntos estratégicos con las mismas técnicas que usaría después en las islas del Paraná y empleó jeroglíficos mayas para tomar notas y transmitir información de manera infranqueable y hermética, sin levantar sospechas dada su profesión de arqueólogo. Durante este tiempo se transformó en un verdadero jefe de espías, produciendo detallados informes secretos sobre los gobiernos y la situación política de los países latinoamericanos y sobre las actividades de los enemigos alemanes, sus redes de aliados locales y, por supuesto, los movimientos de sus submarinos (Harris y Sadler 2003).

Aun cuando los dos lugares donde realizó sus investigaciones, el río Paraná y Tierra del Fuego, fueron en la época puntos geopolíticamente destacados, no hemos hallado evidencia directa de actividades de espionaje desarrolladas por los Lothrop en la Argentina. Una de las razones es la falta de trabajos sistemáticos y de documentos desclasificados de las actividades de espionaje en el período de entreguerras, tanto en la Argentina como en el extranjero, en un contexto donde el foco de atención ha sido colocado en la Segunda Guerra (p.ej., Allen 2011) o en figuras destacadas como el arqueólogo y espía británico T. E. Lawrence (Lawrence de Arabia), entre otros profesionales (Richter 2008 y bibliografía allí citada). A pesar de ello, se puede observar en la gestión de la expedición la incidencia, en la década de 1920, de redes de relaciones que notablemente se solapaban con esa cuestionable actividad. Estas redes estaban generalmente asociadas al comercio, las relaciones diplomáticas internacionales, la expansión global de las corporaciones, la academia y la filantropía cultural mediante el apoyo a actividades científicas en el extranjero de fundaciones norteamericanas, como Carnegie o Rockefeller (Rietzler 2011). A lo cual se le suma la influencia de hombres de negocios expatriados, como Apollonio, quienes pueden ser considerados como brokers que actuaban localmente vinculando cada uno de esos mundos, trabajando en ocasiones como informantes o como subagentes de espionaje.
En esas redes se habían movido en forma previa otros arqueólogos, como el mencionado negociador del Museo Heye ante el Museo de La Plata, Marshall Saville, quien había realizado tareas de inteligencia en Europa, o George A. Dorsey, egresado de Harvard y curador del Field Museum, quien ingresó a la ONI bajo el patronazgo del teniente Edward Breck, muy conocido por haber infiltrado con éxito a la comunidad alemana en Argentina y el Brasil en 1917-18 (Browan 2011). En ese sentido, es de destacar que desde la Primera Guerra Mundial, Buenos Aires se había convertido en la sede central en América del Sur para el desarrollo de las actividades de espionaje de Alemania dirigidas por diplomáticos y encargados de filiales comerciales de ese país -en franca competencia con la expansión de las firmas norteamericanas en el Río de la Plata-, que actuaban asociados con prominentes miembros del gobierno (Escudé y Cisneros 2000). En este contexto, para contrarrestar el espionaje alemán, el gobierno norteamericano, mediante la ONI, había ordenado en 1917 establecer una red secreta de agentes en las zonas costeras de la Argentina y el Uruguay, con el objeto de monitorear los movimientos de los barcos primero, y más tarde de los submarinos, en regiones como en los puntos donde Lothrop dirigió las dos expediciones para el Museo Heye, el Delta del Paraná y Tierra del Fuego.

A partir de la profunda investigación de Price (2008) sí se conoce que en 1940 el deber patriótico y el talento de arqueólogo-espía de Lothrop fueron nuevamente demandados por los Estados Unidos. Esta vez en América del Sur, con motivo de la Segunda Guerra Mundial y al servicio del Special Intelligence Service dentro del FBI. Luego de recibir clases de espionaje y entrenamiento en el uso de códigos secretos, tintas invisibles y protocolos de contacto por el FBI en Washington D.C., estuvo varios años en Lima, Perú. Allí su salario fue cubierto por la Carnegie Institution, la Rockefeller Foundation, Harvard University y el FBI, mostrando cómo la investigación académica, renombradas instituciones, fundaciones y universidades eran interceptadas por agencias estatales de inteligencia y misiones militares (véanse más ejemplos en Price 2008 y Richter 2008). En este caso, Lothrop contó con la ayuda de su segunda esposa, Eleanor Bachman, quien, con su trabajo como periodista, colaboraba en el armado de la "pantalla arqueológica" para cubrir las actividades de espionaje de Samuel y también en la recopilación velada de datos entre sus principales 
entrevistados en el mundo social de Lima. Con el fin de recopilar información sobre las operaciones del Eje en Sudamérica y sobre los movimientos económicos y políticos de Perú, durante cuatro años Lothrop envió a su país sucesivos informes, cartas y telegramas codificados hasta que la policía peruana comenzó a investigar sus actividades, haciéndose cada vez más evidente el hecho de que además de estudiar colecciones de objetos arqueológicos estaba efectuando tareas de espionaje para el FBI.

\section{Consideraciones Finales}

Con el fin de reconstruir el contexto sociohistórico donde tuvo lugar la expedición dirigida por Samuel Lothrop en el Delta del Paraná en 1925, en consonancia con el estudio actual de las colecciones extraídas en esa ocasión y depositadas en el Museo de La Plata y en el Museum of American Indian (Bonomo 2013; Bonomo et al. 2009; Bonomo et al. 2011), en este trabajo hemos analizado de manera somera una serie de episodios vinculados a la misma. Estos episodios muestran las implicancias éticas, económicas y políticas de la práctica arqueológica y la política institucional en el presente. Entre ellos se destaca el convenio de intercambio de colecciones y cooperación establecido por Luis María Torres y George Heye, en un contexto donde el director del Museo de La Plata, para conseguir apoyo económico extra para la gestión institucional, había iniciado una política de relación con acaudalados mecenas locales vinculados en algunos casos a importantes empresas y negocios agropecuarios. Por otro lado, el convenio con el Museum of American Indian se dio en un momento de auge en la expansión de las firmas norteamericanas en la Argentina en fuerte competencia con el dominio de sus homólogas alemanas, donde empresarios al estilo de Heye invirtieron fuertemente en actividades relacionadas con el petróleo y sus derivados, los importantes préstamos financieros al gobierno, e inundando el mercado local con automóviles, maquinarias agrícolas y equipamiento de oficinas. La expedición arqueológica al Delta del Paraná fue uno de los correlatos científicos de esa expansión económica fuera de las fronteras estadounidenses.

En otro orden de cosas, el diario llevado por Lothrop nos permite apreciar, en primer lugar, cómo funcionaban en la práctica las regulaciones establecidas en 1921 para la ley 9080 . Las periódicas visitas de Torres para fiscalizar las excavaciones, la gestión de los permisos y el control de lo extraído, definiendo lo que podía ser enviado a EE.UU. de lo que debía permanecer en el país, parecen abonar lo que hemos señalado en otra parte, referido a que la reglamentación de dicha ley estuvo orientada, antes que a la supervisión de las actividades de los coleccionistas argentinos, al férreo control de las actividades de las expediciones extranjeras y de los objetos que pretendían "exportar" (Farro et al. 2012). En este contexto, es de destacar en segundo lugar la habilidad de Lothrop para agilizar el inicio de los trabajos y hacer más eficiente la gestión de la expedición, por medio de una nutrida red de contactos personales.

No hemos hallado evidencia directa de posibles actividades de espionaje de Lothrop durante sus trabajos de campo en la Argentina. Aun así, las dos regiones litorales trabajadas por Lothrop poseen una importancia geopolítica general para América del Sur. Además, existen pruebas claras de momentos previos y posteriores a sus trabajos en el Delta del Paraná, en los que este investigador utilizó la arqueología para ponerse al servicio de propósitos militares y defender los intereses políticos de su país en territorio latinoamericano. En ese sentido, resulta sugestivo que las redes de relaciones políticas y económicas en las que se apoyó para llevar adelante la expedición, así como las gestiones previas realizadas con Torres por emisarios-espías como Marshall Saville, se solapan notablemente con las complejas redes e infraestructuras atravesadas por la diplomacia, los servicios de espionaje, la competencia comercial internacional, la filantropía cultural de las fundaciones norteamericanas y los vínculos de este entramado con funcionarios y brokers locales.

Agradecimientos: Agradecemos las observaciones y sugerencias realizadas al manuscrito por tres evaluadores anónimos. A Gustavo Politis y Ricardo Bonini por la bibliografía conseguida. A Heather A. Shannon y Lou Stancari (NMAI) por las fotografías facilitadas de los trabajos de campo en El Cerrillo. A Pat Kervick, Jessica Desany Ganong y Viva Fisher (PMAE) por la colaboración durante la consulta del archivo Lothrop y por las gestiones para la publicación de la figura 2. A David H. Price por el asesoramiento de fuentes, archivos y literatura referida a la relación entre arqueología y espionaje. A Diego Gobbo por la confección del mapa. 


\section{Referencias Citadas}

Allen, S.H. 2011. Classical Spies. American Archaeologists with the OSS in World War II Greece. University of Michigan Press, Michigan.

Bonomo, M. 2013. Reanálisis de la colección de Samuel Lothrop procedente del Delta del Paraná. Relaciones de la Sociedad Argentina de Antropología 38(1):169-198.

Bonomo, M., I. Capdepont, y A. Matarrese 2009. Alcances en el estudio de colecciones. Los materiales arqueológicos del Delta del río Paraná depositados en el Museo de La Plata (Argentina). Revista de Arqueología Sudamericana 5:68-101.

Bonomo, M., G. Politis y C. Gianotti 2011. Montículos, jerarquía social y horticultura en las sociedades indígenas del Delta del río Paraná (Argentina). Latin American Antiquity 22:297-333.

Browman, D.L. 2011. Spying by American archaeologists in World War I, with a minor linkage to the development of the Society for American Archaeology. Bulletin of the History of Archaeology 21:2-17.

Easby, D.T. 1965. Samuel Kirkland Lothrop, 1892-1965. American Antiquity 31:256-261.

Endere, M.L. 2000. Arqueología y Legislación en Argentina. Cómo Proteger el Patrimonio Arqueológico. Serie Monográfica INCUAPA, vol. 1, Departamento de Publicaciones UNCPBA, Tandil.

Endere, M.L. e I. Podgorny 1997. Los gliptodontes son argentinos. La ley 9080 y la creación del patrimonio arqueológico nacional. Ciencia Hoy 7(42):54-59.

Escudé, C. y A. Cisneros 2000. Historia de las Relaciones Exteriores de la República Argentina. http://www.argentina-rree. com/historia.htm

Farro, M. 2009. La Formación del Museo de La Plata. Coleccionistas, Comerciantes, Estudiosos y Naturalistas Viajeros a fines del Siglo XIX. Prohistoria editores, Rosario.

Farro, M., S. García y A. Martínez 2012. Expediciones, colecciones y formas de registro. La colección arqueológica Banjamín Muniz Barreto. En Los Secretos de Barba Azul: Fantasías y Realidades de los Archivos del Museo de La Plata, editado por T. Kelly e I. Podgorny, pp. 125-170. Prohistoria ediciones, Rosario.

García, S.V. 2010. Enseñanza Científica y Cultura Académica. La Universidad de La Plata y las Ciencias Naturales (19001930). Prohistoria ediciones, Rosario.

Harris, C.H. y L.R. Sadler 2003. The Archaeologist Was A Spy: Sylvanus G. Morley and the Office of Naval Intelligence. University of New Mexico Press, Albuquerque.

Harvard Alumni Association 1926. "Kenneth Apollonio". Harvard Alumni Bulletin 28(22):36.

Jacknis, I. 2006. A new thing? The NMAI in historical and institutional perspective. The American Indian Quarterly 30(3-4):511-542.
Lanciotti, N. y A. Lluch 2009. Foreign direct investment in Argentina: Timing of entry and business activities of foreign companies (1860-1950). Enterprises et Historie 1(54):37-66.

Lothrop, S.K. 1925a. The Thea Heye La Plata expedition. Indian Notes 2(4):257-266.

- - - 1925b. Museum expedition to Tierra del Fuego. Indian Notes 2(4):322-334.

- - - 1932. Indians of the Paraná Delta, Argentina. Annals of the New York Academy of Science 32:77-232.

- - - 1957. George Gustav Heye. 1874-1956. American Antiquity 23:66-67.

Mac Mullen, A. 2009. Reinventing George Heye. Nationalizing the Museum of American Indian and its collections. En Contesting Knowledge. Museums and Indigenous Perspectives, editado por S. Sleeper-Smith, pp. 65-105. University of Nebraska Press, Lincoln and London.

Márquez Miranda, F. 1937. Doctor Luis María Torres. Revista del Museo de La Plata, (N.S) Sección Oficial:1-10.

Pegoraro, A.S. 2009. Las Colecciones del Museo Etnográfico de la Universidad de Buenos Aires: Un Episodio en la Historia del Americanismo en la Argentina, 1890-1927. Tesis doctoral, Facultad de Filosofía y Letras-UBA, Buenos Aires.

Podgorny, I. 2005. Bones and devices in the constitution of palaeontology at the end of the Nineteenth Century. Science in Context 18:249-283.

Price, D.H. 2008. Anthropological Intelligence. The Deployment and Neglect of American Anthropology in the Second World War. Duke University Press, Durham, London.

Rietzler, K. 2011. Before the Cultural Cold Wars: American philanthropy and cultural diplomacy in the inter-war years. Historical Research 84(223):148-164.

Richter, T. 2008. Espionage and near Eastern archaeology: A historiographical survey. Public Archaeology 7(4):212-240.

Snead, J.E. 1999. Science, commerce, and control: Patronage and the development of anthropological archaeology in the Americas. American Anthropologist 101:256-271.

Thorne, R.C. y R.N. Thorne. 1995. Letters Home from the Bone Camps. Annals of a Field Museum Paleontologist, Argentina and Bolivia, 1926-1927. Privately published, New Mexico.

Torres, L.M. 1911. Los Primitivos Habitantes del Delta del Paraná. Biblioteca Centenaria 4, Universidad Nacional de La Plata, Buenos Aires.

Vignati, M.A. 1941. Censo de paquetes óseos de origen guaraní. Revista del Museo de La Plata 2(9):1-11.

Willey, G. 1976. Samuel Kirkland Lothrop. En Biographical Memoirs, editado por National Academy of Sciences, pp. 253272. The National Academies Press, Washington D.C. 


\section{Notas}

$1 \quad$ Carta de Randolph. M. Saville a L. Ma. Torres, Buenos Aires, 1 de junio de 1922, AHMLP, IMD, carpeta $\mathrm{N}^{\circ} 21$, carta $\mathrm{N}^{\circ} 375$. Por el canje, el Museo Etnográfico de Buenos Aires recibió 353 piezas etnográficas y arqueológicas del Museum of American Indian (Pegoraro 2009).

2 Carta de Marshall H. Saville a L. Ma. Torres, New York, 3 de julio de 1922, AHMLP, IMD, carpeta $\mathrm{N}^{\circ} 25$, carta $\mathrm{N}^{\circ} 159$.

3 Carta de George Gustav Heye a L. Ma. Torres, Buenos Aires, 25 de agosto de 1924, AHMLP, IMD, carpeta $\mathrm{N}^{\circ} 25$, carta $\mathrm{N}^{\circ} 113$.

4 Carta de Samuel K. Lothrop a Luis Ma. Torres, 28 de agosto de 1924, AHMLP, IMD, carpeta No 27, carta No 215 .

5 Carta de L. Ma. Torres a Mrs. Thea Heye, 2 de septiembre de 1924, LC, N XIII, folio 126.

6 Los permisos se conceden finalmente, de manera efectiva a comienzos de abril. Carta del Ministro de Instrucción Pública a L. Ma. Torres, Buenos Aires, 30 de marzo de 1925, AHMLP, IMD, carpeta No 27, carta No 291.

7 "Reglamentación de la ley 9080 sobre protección de yacimientos arqueológicos, antropológicos, paleontológicos y paleoantropológicos, Buenos Aires, 23 de diciembre de 1921”, AHMLP, IMD, carpeta No 38, carta No 189.

8 Carta modelo de L. Ma. Torres para enviar a los gobernadores de Chubut, Chaco, Río Negro, Santa Cruz y La Pampa, La Plata, 24 de septiembre de 1925, AHMLP, LC, No XIII, folio 404.

9 Carta de Robert Lehmann-Nitsche al Ministro de Instrucción Pública, Dr. Antonio Segarna, La Plata, 21 de marzo de 1925, AHMLP, LC No XIII, folio 276.

10 Cf. Carta de S. K. Lothrop a L. Ma. Torres, 22 de marzo de 1925, AHMLP, IMD, carpeta No 27, carta No 257.
11 Riggs utilizaba para el envío de correspondencia, pago de giros y el envío de colecciones, los servicios de Mr. G.C. Withney, manager de la empresa frigorífica Swift del Plata \& Co. Cf. Carta de Elmer Riggs a L. Ma. Torres, 24 de mayo de 1923, AHMLP, IMD, carpeta No 22, carta No 123.

12 Carta de George G. Heye a L. Ma. Torres, Buenos Aires, 25 de agosto de 1924, AHMLP, IMD, carpeta $N^{\circ} 25$, carta $\mathrm{N}^{\mathrm{o}} 113$.

13 La información de esta sección proviene principalmente del diario y las notas de campo de Lothrop: "S.A. - 1925. Mrs. Thea Heye-La Plata Expedition 1925”, Peabody Museum Archive, S.K. Lothrop papers, Call Number 996-27; 1-15, Field Notes/Journals, Folder 9: Argentina.

14 Informe elevado por Pablo Gaggero a L. Ma. Torres sobre expedición al Delta del Paraná, Arroyo Malo, La Plata, 5 de marzo de 1925, División Antropología MLP catálogo No 1925-6397/6416.

15 Carta de José Buroni a S. K. Lothrop, Buenos Aires, 9 de abril de 1925, Lothrop Archive 996-27; 1-15, Field Notes/ Journals. Folder 9: "Argentina".

16 Cartas de E. Tricerri a L. Ma. Torres, Tigre, 11 de mayo, 22 de julio, 12 y 28 de agosto de 1925, y 30 de mayo de 1926 AHMLP, IMD, Carpeta $\mathrm{N}^{\circ} 27$, cartas $\mathrm{N}^{\circ} 409,410$, 411, 412 y 421, respectivamente.

17 Informe elevado por Pablo Gaggero a L. Ma. Torres sobre expedición al Delta del Paraná, Arroyo Malo, La Plata, 5 de marzo de 1925, División Antropología MLP catálogo $\mathrm{N}^{\circ} 1925-6397 / 6416$.

18 Cf. Informe de L. Ma. Torres elevado a F.P. Moreno sobre expediciones al Delta, La Plata, 6 de noviembre de 1905, AHMLP, IMD, carpeta $N^{\circ} 42$, carta $\mathrm{N}^{\circ} 314$. 
